\title{
Jamming Effectiveness Evaluation Based on Radar Detection Probability
}

\author{
Zhanqiang Liu ${ }^{1}$, Lujiang Liang ${ }^{1}$, Chunyang Wang ${ }^{1}$ and Jinshuai Yang ${ }^{1+}$ \\ ${ }^{1}$ Air and Missile Defense College, Air Force Engineering University, Xi'an 710051, China
}

\begin{abstract}
Aimed at the problem that it's difficult to evaluate oppressive jamming effectiveness in the combat of electronic countermeasure, an evaluation method of jamming effectiveness based on radar detection probability was proposed. Combined with radar distance formula and jamming equation, the models of radar detection probability with jamming and without jamming were established respectively. After imitating the combat scenarios of self-defense jamming and support jamming, setting radar parameters and jammer parameters, the changes of radar detection probability of three targets with different radar cross section (RCS) were simulated and analyzed in the pattern of oppressive jamming. The results show that oppressive jamming could reduce radar detection distance and detection probability, decline radar detection performance largely.
\end{abstract}

Keywords: oppressive jamming; jamming effectiveness; detection probability

\section{Introduction}

Oppressive jamming is one of the main jamming patterns in the field of electronic countermeasures, which mainly utilizing the equipment of electronic countermeasures, launching jamming signal, disturbing the normal work process of radar, and achieving the purpose of destroying radar detection performance. Therefore, it's an important means of measuring radar detection ability to evaluate the jamming effectiveness of oppressive jamming accurately.

The research of jamming effectiveness evaluation based on radar detection range has achieved good achievement. References [1-4] studied the changes of radar detection range in the condition of oppressive jamming, eventually analysed and evaluated the jamming effectiveness. References [5] combined radar distance formula and jamming equation, putting forward two kinds of jamming suppression factor to evaluate jamming effectiveness. References [6-7] analysed jamming effectiveness based on the antenna gain of radar. The above-mentioned literatures are lack of considering the influence on radar detection probability from oppressive jamming, and there is not research on radar detection probability as the indicator of jamming effectiveness evaluation [8] yet.

In view of this, this paper put forward the method of jamming effectiveness evaluation based on radar detection probability. Through establishing the model of radar detection probability with or without jamming, to compare and study the changes of radar detection probability in self-defense jamming condition and support jamming condition, and then to evaluate the effectiveness of oppressive jamming.

\section{The model based on detection probability}

\subsection{Signal-to-noise ratio (SNR)}

Radar detection distance without jamming [9] is

\footnotetext{
+ Corresponding author. Liu Zhanqiang. Tel.: +15594999820

E-mail address:15594999820@qq.com.
} 


$$
R_{\mathrm{T}-\max }=\left[\frac{P_{\mathrm{t}} G_{\mathrm{t}} G_{\mathrm{r}} \sigma \lambda^{2}}{(4 \pi)^{3} k T_{0} B_{\mathrm{n}} F_{\mathrm{n}} L_{\mathrm{s}}(S / N)_{\min }}\right]^{1 / 4}
$$

In equation (1), the specific meaning of different parameters is:

$P_{\mathrm{t}}$ : The power of the radar transmitter; $G_{\mathrm{t}}$ : The gain of the radar send antenna; $G_{\mathrm{r}}$ : The gain of the radar receive antenna; $\sigma$ : Radar cross section of target; $\lambda$ : Wavelength of the electromagnetic wave; $k$ :Boltzmann constant; $T_{0}:$ Receive temperature; $B_{\mathrm{n}}$ : Noise bandwidth; $F_{\mathrm{n}}:$ Noise coefficient; $L_{\mathrm{s}}$ : Radar system loss; $(S / N)_{\min }$ : The sensitivity of radar receiver.

Equation (1) is transformation for signal-to-noise ratio (SNR) [8], namely,

$$
\frac{S}{N}=\frac{P_{\mathrm{t}} G_{\mathrm{t}} G_{\mathrm{r}} \sigma \lambda^{2}}{(4 \pi)^{3} k T_{0} B_{\mathrm{n}} F_{\mathrm{n}} L_{\mathrm{s}} R_{\mathrm{T}-\max }^{4}}
$$

\subsection{Signal-to-jamming ratio (SJR)}

Radar detection distance with jamming [2] [6] is

$$
R_{\mathrm{J}-\max }=\left\{\frac{P_{\mathrm{t}} G_{\mathrm{t}} G_{\mathrm{r}} \sigma R_{\mathrm{j}}^{2}}{4 \pi P_{\mathrm{j}} G_{\mathrm{j}} G_{\mathrm{r}}(\theta) T_{\mathrm{j}} \gamma_{\mathrm{j}}(S / J)_{\text {min }}} \times \frac{\Delta f_{\mathrm{j}}}{\Delta f_{\mathrm{r}}}\right\}^{\frac{1}{4}}
$$

$R_{\mathrm{j}}$ : The distance between radar and the jammer; $P_{\mathrm{j}}$ : Radiation power of jammer; $G_{\mathrm{j}}$ : Antenna gain of jammer; $G_{\mathrm{r}}(\theta)$ : Receiving gain of radar under the jamming; $\theta$ : The angle between the line of target and the line of radar to jammer; $T_{\mathrm{j}}$ : Jammer loss; $\gamma_{\mathrm{j}}$ : Polarization Loss; $(S / J)_{\min }$ : The minimal SJR; $\Delta f_{\mathrm{j}}$ : Jamming bandwidth; $\Delta f_{\mathrm{r}}$ : Transmit signal bandwidth of radar.

Equation (3) is transformation for SJR [8], namely,

$$
\frac{S}{J}=\frac{P_{\mathrm{t}} G_{\mathrm{t}} G_{\mathrm{r}} \sigma R_{\mathrm{j}}^{2}}{4 \pi P_{\mathrm{j}} G_{\mathrm{j}} G_{\mathrm{r}}(\theta) T_{\mathrm{j}} \gamma_{\mathrm{j}} R_{\mathrm{J}-\max }^{4}} \times \frac{\Delta f_{\mathrm{j}}}{\Delta f_{\mathrm{r}}}
$$

In equation (4), when the radar is under the jamming, it's receiving gain [1] [8] [10] is:

$$
G_{\mathrm{r}}(\theta)=\left\{\begin{array}{l}
G_{\mathrm{r}},|\theta| \leq \theta_{0.5} / 2 \\
K\left(\theta_{0.5} / \theta\right) G_{\mathrm{r}}, \theta_{0.5} / 2<|\theta| \leq 90^{\circ} \\
K\left(\theta_{0.5} / 90^{\circ}\right) G_{\mathrm{r}}, 90^{\circ}<|\theta| \leq 180^{\circ}
\end{array}\right.
$$

Where: $\theta_{0.5}$ is radar horizontal beam width; $K$ is a constant, usually $0.04 \sim 0.1$, and $\mathrm{K}=0.1$ in simulation.

When noise jamming is adopted, jamming and system noise [8] will be added:

$$
\frac{S}{N+J}=\frac{1}{1 /(S / N)+1 /(S / J)}
$$

\subsection{Detection probability}

The probability of false alarm defined as the probability that a sample of targets, $\mathrm{R}$ of the signal $r(t)$ will exceed the threshold voltage when noise alone is present in the radar. The formulation [11] is represented as:

$$
\begin{gathered}
P_{\mathrm{fa}}=\int_{V_{\mathrm{T}}}^{\infty} \frac{r}{\sigma_{n}^{2}} \exp \left(-\frac{r^{2}}{2 \sigma_{n}^{2}}\right) d r=\exp \left(-\frac{V_{\mathrm{T}}^{2}}{2 \sigma_{n}^{2}}\right) \\
V_{\mathrm{T}}=\sqrt{2 \sigma_{n}^{2} \ln \left(1 / P_{\mathrm{fa}}\right)}
\end{gathered}
$$

Detection probability $P_{\mathrm{d}}$ is the probability that a sample of targets, $R$ of $r(t)$ will exceed the threshold voltage in the presence of noise and target signal. This is expressed as: 


$$
P_{\mathrm{d}}=\int_{V_{\mathrm{T}}}^{\infty} \frac{r}{\sigma_{n}^{2}} I_{0}\left(\frac{r A}{\sigma_{n}^{2}}\right) \exp \left(-\frac{r^{2}+A^{2}}{2 \sigma_{n}^{2}}\right) d r
$$

Given the assumption that the signal from the radar has the form of a sine wave with amplitude, $A$ and power $=A^{2} / 2$,

$$
\mathrm{SNR}=\frac{A^{2}}{2 \sigma_{n}^{2}}
$$

The equation of detection probability becomes [11]:

$$
\begin{aligned}
& P_{\mathrm{d}}=\int_{\sqrt{2 \sigma_{n}^{2} \ln \left(1 / P_{\mathrm{fa}}\right)}}^{\infty} \frac{r}{\sigma_{n}^{2}} I_{0}\left(\frac{r A}{\sigma_{n}^{2}}\right) \exp \left(-\frac{r^{2}+A^{2}}{2 \sigma_{n}^{2}}\right) d r \\
& =Q\left[\sqrt{A^{2} / \sigma_{n}^{2}}, \sqrt{2 \ln \left(1 / P_{\mathrm{fa}}\right)}\right] \\
& =Q\left[\sqrt{2 \mathrm{SNR}}, \sqrt{-2 \ln \left(P_{\mathrm{fa}}\right)}\right]
\end{aligned}
$$

Where $Q$ known as Marcum's $Q$-function [11-12] and expressed as:

$$
Q[\alpha, \beta]=\int_{\beta}^{\infty} \varsigma I_{0}(\alpha \varsigma) e^{-\left(\varsigma^{2}+\alpha^{2}\right)} d \varsigma
$$

North approximated detection probability [11] [13] as:

$$
P_{\mathrm{d}}=\frac{1}{2}\left[1+\operatorname{erf}\left(\sqrt{\frac{1}{2}+\frac{S}{N}}-\sqrt{\lg \left(\frac{1}{P_{\mathrm{fa}}}\right)}\right)\right\rfloor
$$

The complementary error function erf is expressed as:

$$
\operatorname{erf}(x)=\frac{2}{\sqrt{\pi}} \int_{0}^{x} e^{-u^{2}} d u
$$

Combined with equation (6) and equation (13), when the radar will be in jamming conditions, detection probability with jamming is:

$$
P_{\mathrm{d}}=\frac{1}{2}\left\lfloor 1+\operatorname{erf}\left(\sqrt{\frac{1}{2}+\frac{S}{N+J}}-\sqrt{\lg \left(\frac{1}{P_{\mathrm{fa}}}\right)}\right)\right\rfloor
$$

\section{Simulation and analysis}

\subsection{Parameter settings}

Radar parameter settings are as follows:

Table 1 Radar parameters setting

\begin{tabular}{cc}
\hline Radar parameters & Values \\
\hline Transmit Power/kw & 100 \\
Antenna Gain/dB & 35 \\
Boltzmann constant $/ \mathrm{J} / \mathrm{K}$ & $1.38 \times 10^{-23}$ \\
Receive temperature $/ \mathrm{K}$ & 290 \\
Receive bandwidth $/ \mathrm{MHz}$ & 5 \\
Noise Coefficient/dB & 3 \\
System Loss/dB & 5 \\
The probability of false alarm & $1 \times 10^{-6}$ \\
\hline
\end{tabular}

Jammer parameter settings are as follows:

Table 2 Jammer parameter setting

\begin{tabular}{cc}
\hline Jammer parameters & Values \\
\hline Jamming Power/w & 100 \\
Jamming Antenna Gain/dB & 10 \\
Jamming Band Width/MHz & 20 \\
Polarization Loss & 0.5 \\
Jammer Loss/dB & 4 \\
\hline
\end{tabular}




\subsection{Self-defense jamming}

Considering scenario shown in figure 1.

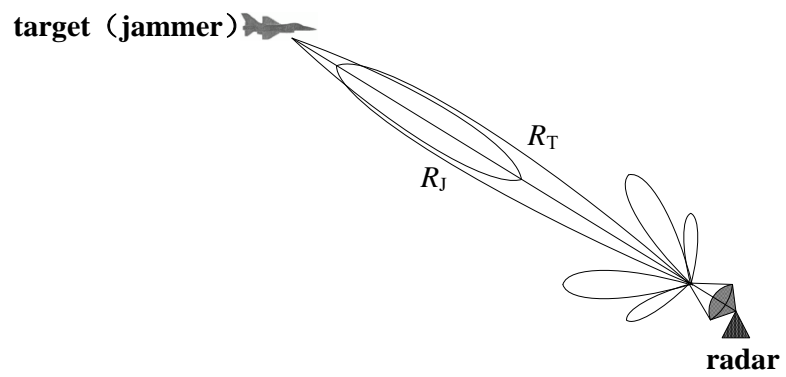

Fig. 1: Self-defense jamming scenarios

The target airplane released the jamming signal to protect its own safety after detecting the threat from the ground radar. The jamming signal mainly entered the main lobe of radar, suppressing and shrinking radar detection range, reducing radar detection performance, and enhancing its own penetration survivability.

On the basis of the above operational scenarios, assumed that the target RCS is $10 \mathrm{dBsm}, 15 \mathrm{dBsm}$ and $20 \mathrm{dBsm}$ respectively, and combined with equation (2), the SNR change of three targets in the different space distance would be got, it is showed in fig 2 .

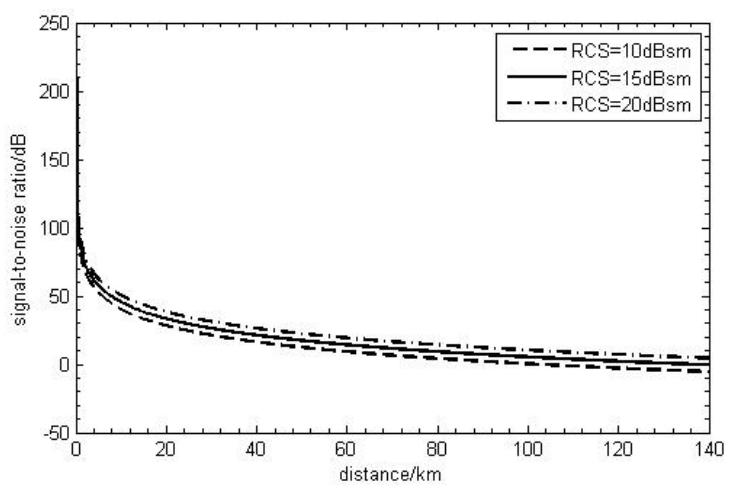

Fig. 2: Signal-to-noise ratio of the different distance

From fig.2, the SNR change range of three targets is $-10 \mathrm{~dB} \sim 250 \mathrm{~dB}$ on the different distances. And at the same distance, the smaller the target RCS would be, the lower SNR is. Besides, SNR will be lower when the space distance gets farther.

Combined with equation (2) and equation (13), Radar detection probability of three kinds of targets could be simulated and calculated on the different distances. The result is showed in Fig.3.

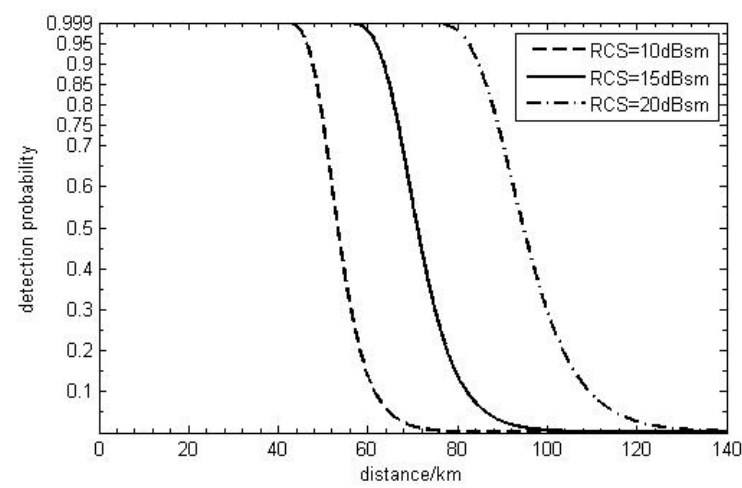

Fig. 3: Radar detection probability of the different distance in the absence of jamming condition

As shown in Fig. 3, when the target RCS is 10dBsm, the maximum detection range of radar is about $47.5 \mathrm{~km}$; when the target RCS is $15 \mathrm{dBsm}$, the maximum detection range of radar is about $63.4 \mathrm{~km}$; when the target RCS is $20 \mathrm{dBsm}$, the maximum detection range of radar is about $84.6 \mathrm{~km}$. Obviously, the smaller the target RCS is, the shorter the maximum detection of radar would be, and radar detection range gets smaller.

Analyzing equation (3), jamming type mainly is self-defense jamming and escort jamming when $G_{\mathrm{r}}(\theta)$ is equal to $G_{\mathrm{r}}$, namely, the jamming signal enters the main lobe of radar, so Fig.4 is showed 
responding the SJR change of the different distance. Making use of the function relationship of SJR and detection probability, Radar detection probability with jamming was simulated, as Fig.5 showed.

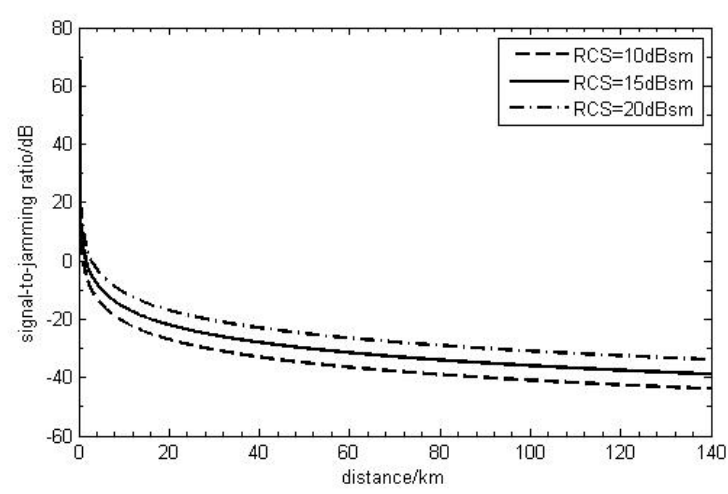

Fig. 4: Signal-to-jamming ratio of the different distance

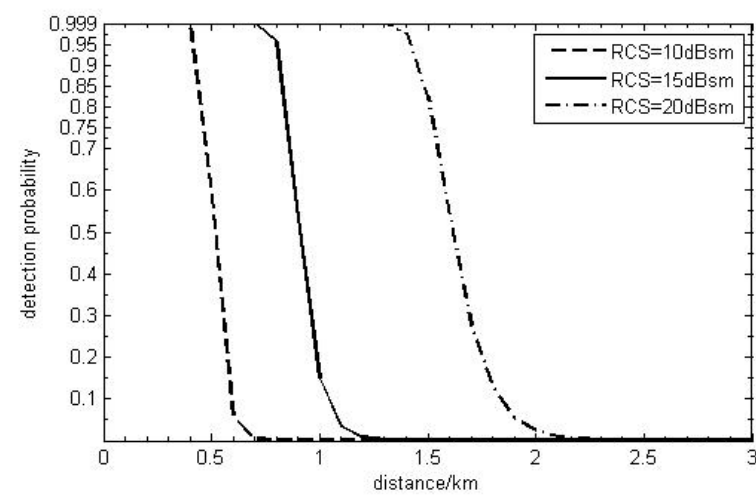

Fig.5 Radar detection probability of the different distance in jamming condition

From the analysis of Fig.4, the SJR change of three targets is $-50 \mathrm{~dB} \sim 70 \mathrm{~dB}$ on the different distances. And the SJR is decreasing function of the distance. And Fig.5 indicated that radar detection probability of three targets is reduced obviously, and due to jamming signal into the main lobe of radar, at this time radar detection probability is mainly on the direction of radar main lobe. Apparently, it's possible to achieve the jamming purpose when the jamming distance of targets will be about $3 \mathrm{~km}$. In a word, the target can not only protect itself in self-defense jamming condition, but also can cover other targets in escort-support jamming condition.

Comparing and analyzing Fig. 3 and Fig.5, which can draw the following conclusions:

1) When jamming signal entered the main lobe of radar, jamming effectiveness is the best, and the smaller the target RCS is, the better jamming efficacy would be.

2) Targets with self-defense jamming condition, radar detection distance reduced three orders of magnitude. Significantly, self-defense jamming could reduce radar detection range, and decline radar detection probability.

\subsection{Support jamming}

Considering scenario shown in figure 6.

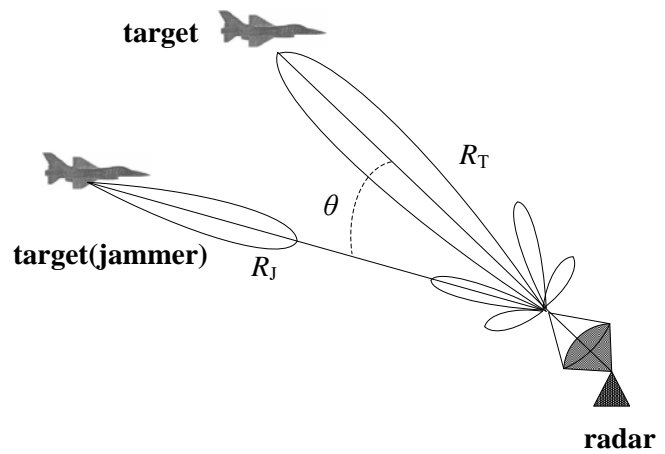

Fig. 6: Support jamming scenarios

The target airplane would face the threat from the ground radar when entering radar detection region, at this moment the airplane with electronic jamming equipment operated with target airplane together.it is under the premise that ensure its own safety, for the purpose of covering target airplane, releasing the jamming signal into the side lobe of radar or the main lobe of radar, which could reduce radar detection performance, and improve the penetration ability of the target airplane.

In order to research the effect of support jamming, according to equation (5), assumed that $\theta_{0.5}$ is equal to 3 degrees, when the radar is under the jamming, its receiving gain of different jamming directions is shown in Fig.7. 


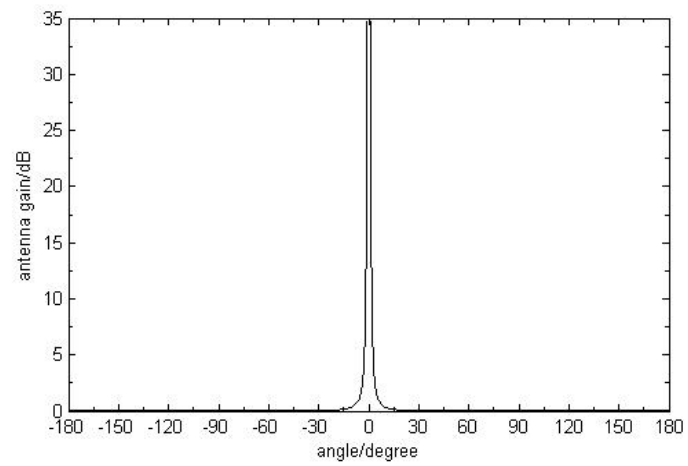

Fig.7: Radar receiving gain of different jamming directions

From fig.7, when jamming angle is around 0 degrees, radar receiving gain is the largest, and gain is main lobe of radar at this moment. But in the other direction of jamming, receiving gain is very small, almost close to 0 degrees.

On the basis of radar receiving gain of different jamming directions, combined with radar detection probability formula of jamming condition, obtained radar detection probability of three targets on different angles when making sure the corresponding jamming distance after setting detection probability is less than 0.9, 0.5and 0.1 respectively. Fig. 8 (a) is the simulation result that target RCS is $10 \mathrm{dBsm}$; Fig. 8 (b) is the simulation result that target RCS is $15 \mathrm{dBsm}$; and Fig. 8 (c) is the simulation result of $20 \mathrm{dBsm}$.

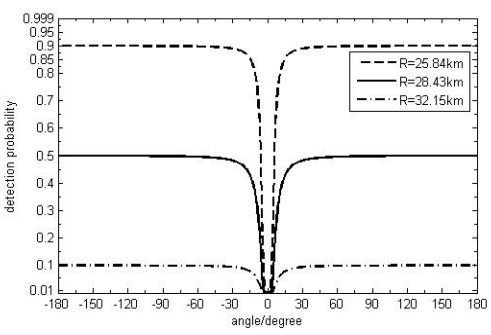

(a) $\mathrm{RCS}=10 \mathrm{dBsm}$

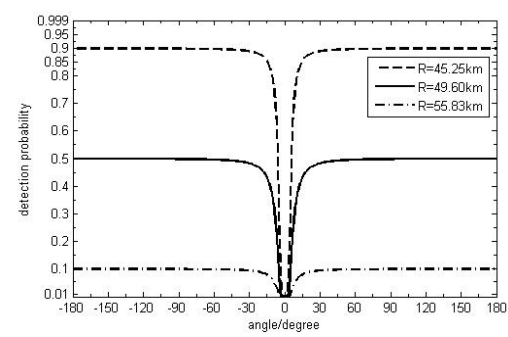

(b) $\mathrm{RCS}=15 \mathrm{dBsm}$

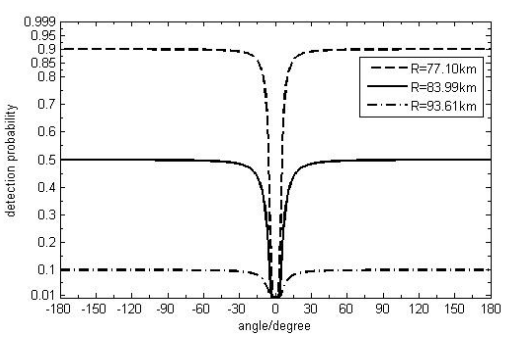

(c) $\mathrm{RCS}=20 \mathrm{dBsm}$

Fig. 8: Radar detection probability of different jamming directions

As fig. 8 showed, when jamming angle is around 0 degrees, radar detection probability is the lowest no matter how far jamming distance is. In the other angles of jamming, detection probability of jamming condition is lower than it is not in jamming condition. In addition, the smaller target RCS is, the shorter jamming distance that is to achieve the established detection probability will be. The results indicated that jamming distance and direction would have an important effect on jamming efficiency. In other words, when jamming signal enters the main lobe of radar, and the shorter jamming distance will be, the better the jamming efficiency is.

For convenience of analysis, table 3 listed radar detection distance without jamming (as showed in Fig.3) when reaching three kinds of detection probability respectively, and table 4 is about detection distance with jamming.

Table 3: Radar detection distance without jamming

\begin{tabular}{cccc}
\hline $\mathrm{RCS} / \mathrm{dBsm}$ & $P_{\mathrm{d}} \leqslant 0.9$ & $P_{\mathrm{d}} \leqslant 0.5$ & $P_{\mathrm{d}} \leqslant 0.1$ \\
\hline 10 & $\geqslant 47.58 \mathrm{~km}$ & $\geqslant 53.19 \mathrm{~km}$ & $\geqslant 61.50 \mathrm{~km}$ \\
15 & $\geqslant 63.42 \mathrm{~km}$ & $\geqslant 70.95 \mathrm{~km}$ & $\geqslant 81.98 \mathrm{~km}$ \\
20 & $\geqslant 84.57 \mathrm{~km}$ & $\geqslant 94.64 \mathrm{~km}$ & $\geqslant 109.36 \mathrm{~km}$ \\
\hline
\end{tabular}

In table 3 , to achieve the same detection probability that required different radar detection distance when detecting different goal. And the larger the target RCS is, the farther radar detection distance of the same detection probability needed.

Table 4: Radar detection distance with jamming

\begin{tabular}{cccc}
\hline RCS/ dBsm & $P_{\mathrm{d}} \leqslant 0.9$ & $P_{\mathrm{d}} \leqslant 0.5$ & $P_{\mathrm{d}} \leqslant 0.1$ \\
\hline 10 & $\geqslant 25.84 \mathrm{~km}$ & $\geqslant 28.43 \mathrm{~km}$ & $\geqslant 32.15 \mathrm{~km}$ \\
15 & $\geqslant 45.25 \mathrm{~km}$ & $\geqslant 49.60 \mathrm{~km}$ & $\geqslant 55.83 \mathrm{~km}$ \\
20 & $\geqslant 77.10 \mathrm{~km}$ & $\geqslant 83.99 \mathrm{~km}$ & $\geqslant 93.61 \mathrm{~km}$ \\
\hline
\end{tabular}


In table 4, to reach the same detection effect without jamming, jamming distance of jammer is much smaller than when being not in jamming condition.

Through comparing table 3 and table 4, the conclusion is as follows:

1) Cover the same target in the absence of jammer, radar detection distance is far away; when covered by jammer, radar detection range is relatively close; this suggests that the jammer can reduce radar detection distance, and promote the penetration survivability of target.

2) When considering burning through distance, radar detection probability with jamming of target at the same distance is smaller than without jamming.so jammer can decline radar detection probability.

In conclusion, detection distance and detection probability of radar would go down in jamming condition.

\section{Conclusions}

This paper put forward a kind of jamming effectiveness evaluation method based on radar detection probability, and combined with radar formula and jamming equation, setting up two kinds of the model for radar detection probability with jamming and without jamming condition. Through imitating the combat scenarios of self-defense jamming and support jamming, and setting the general system of radar and jammer parameters, to compare and study the changes of radar detection probability for three different RCS of target under two kinds of jamming pattern. The conclusion is as follows:

1) Self-defense jamming can reduce radar detection probability, and decline radar detection performance. And the smaller the target RCS is, the better jamming effectiveness would be.

2) There are differences for radar detection probability in different jamming direction when being in support jamming condition. In the direction of the main lobe of radar, jamming effectiveness is the best and detection probability would be the lowest. In addition, the bigger jamming signal deviating from the main lobe of radar is, the higher detection probability would be, and jamming effectiveness is not ideal. At the same time the smaller the RCS of covering target is, the better jamming efficacy would be.

The above-mentioned study only considered the jamming pattern, but lacking of the further study on the type of jammer and actual combat environment. The following study will pay more attention to the attackdefence process of jammer and radar. Besides, this paper does not take into account the influence of pulse accumulation, the next step will focus on analysing the relationship between radar cumulative detection probabilities and jamming effectiveness.

\section{References}

[1] M. J. Lv, M. Zhou, Q. L. Du. The integrative application of anti-jamming technology for radar. In: M. Zhou, et al (eds.). Proc. of the 2nd International Workshop on Intelligent Systems \& Applications. Wuhan, China: IEEE, 2010, pp.1-4.

[2] H. F. Song, M. Q. Xiao, H. Wu, Y. Chen, Y. W. Ge. Generic model of aircraft susceptibility to different airborne electronic counter measures. Acta Aeronautica et Astronautica Sinica.2015, 36(11):3630- 3639.

[3] B. Xiao, C.S. Sun. Modeling and simulation on radar detection range under complex electromagnetic environment. In: C.S. Sun (eds.). International Conference on Virtual Reality and Visualization. Beijing, China: IEEE, 2011, pp. 315-318.

[4] Z. Yang, S. L. Li, J. J. Yin. Study of aircraft survivability under the conditions of self-defense jamming and chaff jamming. Transactions of Beijing Institute of Technology.2013, 33(4):375-379.

[5] Y. Zhu, C. Gao. Z. L. Wang. Modeling of jamming destroy factor. In: C. Gao, et al (eds.).Proceeding of 2011 4th International Congress on Image and Signal Processing. IEEE, 2011, pp.198-201.

[6] C. Q. Luo, X. L. Li. Research of non-coherent detection in jamming environment .In: X. L. Li. (eds.). Proceeding of the 2013 CIE International Conference on Radar. Xi'an: IEEE, 2013, pp.0610-0610. 
[7] W. Pan, Z. M. Song, L. Ma. Research on force assignment for ground-to-air radar jamming system based on chaos genetic algorithms. In: Z. M. Song, et al (eds.). Proceeding of the Chinese Control \& Decision Conference. Qingdao, China: IEEE, 2015, pp. 1215-1220.

[8] T. Y. Shen, J. J Ding, Y. Ding, J. G. Shi. A method of detection performance modeling in jamming condition based on radar network system. In: J. J Ding, et al (eds.). Proceeding of the 2011 IEEE cie International Conference on Radar. Piscataway. NJ: IEEE, 2011, pp.1366-1369.

[9] L. W. Wang, X. S. Jiang. An improved radar detection range plotting method based on radar equation. In: X. S. Jiang (eds.). Proceeding of the 2011 International Conference on Machine Learning \& Cybernetics. Melbourne, Australia: Machine Learning and Cybernetics, 2011, pp.381-385.

[10] T. Q. Ni, J. D. Wang, Y. A. Liu. Research on Target Route Planning of Confrontation of ESJ to Radar Network. Acta Armamentarh. 2010, 31(12): 1599- 1603.

[11] O. Chiemela, B. Glen. Autonomous marine craft navigation: on the study of radar obstacle detection. In: B. Glen (eds.). Proceeding of the 2011 International Conference on Machine Learning \& Cybernetics. Melbourne, Australia: Machine Learning and Cybernetics, 2011, pp.567-572.

[12] M. O. Mughal, J. R. Gu, J.M. Kim. Detection probability analysis for AF assisted cooperative spectrum sensing in cognitive radio networks In: J. R. Gu, et al (eds.).Proceeding of the International Conference on ICT Convergence. Seoul, South Korea, 2011, pp. 461- 464.

[13] B. R. Mahafza, A. Z. Elsherbeni. MATLAB Simulations for Radar System Design. House of Electronics Industry Press, 2009. 